Supplement of Biogeosciences Discuss., 11, 9421-9449, 2014

http://www.biogeosciences-discuss.net/11/9421/2014/

doi:10.5194/bgd-11-9421-2014-supplement

(C) Author(s) 2014. CC Attribution 3.0 License.

(c) (i)

Supplement of

\title{
Typhoons exert significant but differential impact on net carbon ecosys- tem exchange of subtropical mangrove ecosystems in China
}

H. Chen et al.

Correspondence to: G. Lin (lingh@ mail.tsinghua.edu.cn) 
Table S1. Characteristics of typhoon landed near Yunxiao (YX) and Gaoqiao (GQ) site during a four year period between 2009 and 2012 .

\begin{tabular}{|c|c|c|c|c|c|c|c|}
\hline Name & DOY $_{\text {Land }}$ & Duration & Category & Wind $_{\text {Land }}$ & Wind $_{\text {min. distance }}$ & Distance $_{\min }$ & Rainfall \\
\hline \multicolumn{8}{|l|}{ YX site } \\
\hline Linfa & 171 & 35 & 9 & 23 & 18.66 & 100 & 43.70 \\
\hline Molave & 199 & 16 & 12 & 35 & 18.22 & 223 & 9.10 \\
\hline Lionrock & 245 & 41 & 9 & 23 & 17.58 & 9 & 79.30 \\
\hline Namtheum & 243 & 9 & 8 & 18 & 7.87 & 171 & 8.00 \\
\hline Meranti & 253 & 25 & 12 & 35 & 11.14 & 142 & 3.00 \\
\hline Fanapi & 263 & 32 & 12 & 35 & 23.06 & 23 & 85.8 \\
\hline Megi & 295 & 36 & 13 & 38 & 24.62 & 29 & 84.3 \\
\hline Sarika & 161 & 15 & 8 & 18 & 18.22 & 42 & 20.9 \\
\hline Babj & 241 & 45 & 9 & 23 & 9.86 & 127 & 56.8 \\
\hline \multicolumn{8}{|l|}{ GQ site } \\
\hline Soudelor & 193 & 20 & 8 & 18 & 18.05 & 141 & 0.20 \\
\hline Goni & 217 & 74 & 9 & 23 & 19.47 & 73 & 100.00 \\
\hline Mujigae & 254 & 16 & 8 & 20 & 14.33 & 197 & 31.4 \\
\hline Koppu & 258 & 24 & 12 & 35 & 9.04 & 133 & 95.8 \\
\hline Parma & 285 & 46 & 9 & 23 & 11.74 & 212 & 115.80 \\
\hline Chanthu & 203 & 34 & 12 & 35 & 22.01 & 29 & 16.30 \\
\hline Nockten & 210 & 14 & 10 & 28 & 17.81 & 197 & 2.8 \\
\hline Nesat & 272 & 25 & 12 & 35 & 25.76 & 122 & 55.6 \\
\hline
\end{tabular}


DOY $_{\text {Land: }}$ The time of year that typhoon made landfall;

Duration: The length of time when the typhoon occurred at a distance less than 300 km from our study site;

Category: Beaufort wind force scale;

Wind Land $_{\text {The }}$ maximum wind speed of typhoon when made landfall;

Wind $_{\text {min.distance: }}$ The maximum wind speed near mangrove ecosystem when the typhoon was the nearest to it;

Distance $_{\text {min: }}$ The minimum distance from mangrove study site during typhoon period. 